\title{
MOLECULAR DESIGN OF COLLOIDS IN SUPERCRITICAL FLUIDS
}

Final Report for the period: September 1, 2004 through October 14, 2008

Date Published- April 2009

Keith P. Johnston

PREPARED FOR THE UNITED STATES DEPARTMENT OF ENERGY/OFFICE OF BASIC ENERGY SCIENCES

DOE Grant DE-FG02-04ER15549

Address: The University of Texas at Austin

P.O. Box 7726, Austin, TX 78713-7726 


\section{Summary}

Overview

The environmentally benign, non-toxic, non-flammable fluids water and carbon dioxide $\left(\mathrm{CO}_{2}\right)$ are the two most abundant and inexpensive solvents on earth. Emulsions of these fluids are of interest in many industrial processes, as well as $\mathrm{CO}_{2}$ sequestration and enhanced oil recovery. Until recently, formation of these emulsions required stabilization with fluorinated surfactants, which are expensive and often not environmentally friendly. In this work we overcame this severe limitation by developing a fundamental understanding of the properties of surfactants the $\mathrm{CO}_{2}$-water interface and using this knowledge to design and characterize emulsions stabilized with either hydrocarbon-based surfactants or nanoparticle stabilizers. We also discovered a new concept of electrostatic stabilization for $\mathrm{CO}_{2}$-based emulsions and colloids. Finally, we were able to translate our earlier work on the synthesis of silicon and germanium nanocrystals and nanowires from high temperatures and pressures to lower temperatures and ambient pressure to make the chemistry much more accessible.

\section{Key Advancements in Four Primary Projects}

The following summaries describe highlights of our work in four primary areas. Except for the project, "Electrostatic Stabilization of Colloids in CO2 and Low Dielectric Solvents", the other three were in the original proposal. More detailed results are given in the published papers in the bibliography.

\section{Stabilization of Emulsions of Water and Carbon Dioxide with Hydrocarbon Surfactants}

The interfacial properties, texture, and stabilities of high-pressure carbon dioxide-in-water (C/W) foams (also called emulsions) were studied and analyzed for a series of hydrocarbon surfactants. A novel paradigm has been discovered, based on molecular dynamics computer simulation and interfacial measurements, to design branched ("stubby”) tails for hydrocarbon surfactants with high activity at the water- $\mathrm{CO}_{2}$ interface. These branched tails block the interface between water and carbon dioxide to lower the interfacial tension more effectively than linear surfactants. As a consequence of this better blocking of the interface, the interfacial tension deceases, the surface pressure increases, and the C/W foams become highly stable. For branched surfactants, the stabilities were markedly lower for air/water foams and decane-water emulsions. The greater stability of the $\mathrm{C} / \mathrm{W}$ foams to coalescence has been described in terms of various interfacial properties. These include a smaller capillary pressure, lower drainage rates, and a sufficient 
surface pressure and thus dilational modulus, plus small film sizes, to hinder spatial and surface density fluctuations that lead to coalescence. Unexpectedly, the foams were stable even when the surfactant favored the $\mathrm{CO}_{2}$ phase over the water phase, in violation of conventional models. This unusual behavior is explained in terms of by the low drainage rate, which makes Marangoni stabilization of less consequence, and the strong tendency of emerging holes in the lamella to close as a result of surfactant tail flocculation in CO2. The high distribution coefficient towards $\mathrm{CO}_{2}$ versus water is of significant practical interest for mobility control in $\mathrm{CO}_{2}$ sequestration and enhanced oil recovery by foam formation. It has been estimated that the amount of surfactant that could be utilized in these processes would be comparable to all of the surfactant used in the world for all other processes. Our fundamental research for DOE was enhanced with a grant from the Dow Chemical Company to explore advanced surfactants to design foams for these processes .

\section{Nanoparticle Stabilization of Emulsions of Water and Carbon Dioxide}

In this proposal, we have shown that macroemulsions of water and carbon dioxide may be stabilized with particles instead of surfactants. Hydrophilic particles, such as silica, are wetted more by the water phase leading to a $\mathrm{C} / \mathrm{W}$ emulsion, in which the interface bends around $\mathrm{CO}_{2}$. Conversely, hydrophobic particles are oriented towards the $\mathrm{CO}_{2}$ phase, leading to a W/C emulsion. Changes in the contact angle (wetting) of a solid particle at the $\mathrm{CO}_{2}$-water interface have been accomplished via adjustments in the surface chemistry of particles to control the emulsion morphology.

The stabilization of emulsions with particles in $\mathrm{CO}_{2}$ circumvents the problem of poor solvation of some surfactant tails by $\mathrm{CO}_{2}$. The particles can be thought of as a highly "stubby" surfactant tail that blocks more of the water- $\mathrm{CO}_{2}$ interface than traditional narrower surfactant tails. We have recently been given two grants from the Advanced Energy Consortium, which is composed of 9 industrial companies, to build on this fundamental research to investigate nanoparticle migration through porous media and nanoparticle sensing of interfaces in oil reservoirs.

\section{Electrostatic Stabilization of Colloids in CO2 and Low Dielectric Solvents}

Although numerous studies have reported the behavior of steric stabilization in compressed supercritical fluids including carbon dioxide, the possibility of electrostatic 
stabilization has not been considered due to the exceptionally low values of the dielectric constant $\varepsilon_{r}$, typically 1.5. Any counterions in the double layer would have a strong tendency to ion pair with charges on the surface of the colloid due to the low $\varepsilon_{r}$. However, we were able to achieve electrostatic stabilization by engulfing these counterions in micelles to limit this ion pairing and to study the mechanism both experimentally and theoretically. Electrostatic repulsion was shown to stabilize micrometer-sized water droplets with spacings greater than $10 \mu \mathrm{m}$ in an ultra-low dielectric $\mathrm{CO}_{2}$ at elevated pressures. The counterions, stabilized with a nonionic highly branched stubby hydrocarbon surfactant, form an extremely thick double layer. We developed a new technique, optical coherence tomography, to measure electrophoretic mobilities of $\mathrm{TiO}_{2}$ colloids in apolar solvents at low dielectric constants where typical techniques are not applicable.

\section{High Temperature Synthesis of Germanium Nanocrystals and Nanowires at Atmospheric} Pressure

Semiconductor nanocrystals exhibit size-tunable optical and electronic properties that are of interest in solid state lighting, sensors and other electronic and optoelectronic devices. Prior to this project, solution-phase methods have been developed to produce Si and Ge nanoparticles

and nanowires at high temperatures and pressures (typically $>200$ bar). For Ge nanocrystals, it has been challenging to identify appropriate precursors that readily decompose in the presence of organic ligands at lower temperatures and sustain controlled crystalline particle growth. In this study, we were able to synthesize crystalline nanoparticles and nanowires at atmospheric pressure at $300^{\circ} \mathrm{C}$ by utilizing highly reactive $\mathrm{Ge}(\mathrm{II})$ precursors, along with organic ligands with low volatility, thus making this chemistry far more accessible.

\section{Detailed Abstracts for Selected Projects}

1. High Internal Phase CO2-in-Water Emulsions Stabilized with a Branched Nonionic Hydrocarbon Surfactant

A nonionic-methylated branched hydrocarbon surfactant, octa(ethylene glycol) 2,6,8- trimethyl4-nonyl ether ( $\left.5 b-\mathrm{C}_{12} \mathrm{E}_{8}\right)$ emulsifies up to $90 \% \mathrm{CO}_{2}$ in water with polyhedral cells smaller than $10 \mu \mathrm{m}$, as characterized by optical microscopy. The stability of these concentrated $\mathrm{CO}_{2} /$ water (C/W) emulsions increases with pressure and in some cases exceeds 24 hours. An increase in 
pressure weakens the attractive van der Waals interactions between the $\mathrm{CO}_{2}$ cells across water and raises the disjoining pressure. It also enhances the solvation of the surfactant tail and drives the surfactant from water towards the water- $\mathrm{CO}_{2}$ interface, as characterized by the change in emulsion phase behavior and the decrease in interfacial tension $(\gamma)$ to $2.1 \mathrm{mN} / \mathrm{m}$. As the surfactant adsorption increases, the greater tendency for ion adsorption is likely to increase the electrostatic repulsion in the thin lamellae and raise the disjoining pressure. As pressure increases, the increase in disjoining pressure and decrease in the capillary pressure (due to the decrease in $\gamma$ ) each favor greater stability of the lamellae against rupture. The electrical conductivity is predicted successfully as a function of Bruggeman's model for concentrated emulsions. Significant differences in the stability are observed for concentrated C/W emulsions at elevated pressure versus air/W or C/W foams at atmospheric pressure.

\section{Tertiary Amine Esters for Carbon Dioxide-Based Emulsions}

Tertiary amine esters, a new class of surfactants for $\mathrm{CO}_{2}$-based dispersions, stabilize carbon dioxide-in-water macroemulsions for several hours even at a $\mathrm{CO}_{2}$ density as low as $0.74 \mathrm{~g} / \mathrm{mL}$ (70 bar) at $298 \mathrm{~K}$. The combination of a weakly hydrophilic tertiary amine, which is protonated by carbonic acid, and branched ester tails provides proper values of the Hydrophilic- $\mathrm{CO}_{2}$-philic Balance (HCB) for emulsion stabilization. The surfactant nitrilotripropane-1,2-diyl tripivalate (tBu-TIA) lowered the $\mathrm{CO}_{2}$-water interfacial tension to $2.6 \mathrm{mN} / \mathrm{m}$ as a result of the stubby architecture (low aspect ratio) of the surfactant tail, which helps block contact between water and carbon dioxide. The high level of methylation produces a smaller $\gamma$ and greater emulsion stability relative to nitrilotripropane-1,2-diyl triacetate. Relative to the high-pressure $\mathrm{CO}_{2}$-water system with a $\mathrm{pH}$ of 3.3, an increase in $\mathrm{pH}$ with the addition of $\mathrm{NaOH}$ decreases interfacial activity and reduces emulsion stability, as the surfactant is deprotonated. The adsorption isotherm shows a high interfacial area per surfactant molecule $\left(400 \AA^{2}\right)$ as a result of the stubby structure of the surfactant. The extremely low aspect ratio of this surfactant compared to other hydrocarbon surfactants shields water from $\mathrm{CO}_{2}$ at the interface, resulting in a lower the interfacial tension, and minimizes interactions between surfactant tail groups. These factors make these low-molecular weight amine esters desirable for tunable $\mathrm{CO}_{2}$-in-water emulsions, as a replacement for more widely used fluorinated surfactants. The facile synthesis of a variety of 
tertiary amine esters makes this class of surfactants attractive for developing structure-property relationships

3. Wetting phenomena at the CO2/Water/Glass Interface

A novel high-pressure apparatus and technique were developed to measure $\mathrm{CO}_{2} /$ water/solid contact angles (As) in situ for pressures up to 204 bar. For two glass substrates with different hydrophilicities, $\theta$ increased significantly with $\mathrm{CO}_{2}$ pressure. As pressure was increased, an increase in the cohesive energy density of $\mathrm{CO}_{2}$ caused the substrate- $\mathrm{CO}_{2}$ and water- $\mathrm{CO}_{2}$ interfacial tensions $(\gamma \mathrm{s})$ to decrease, while the water-substrate $\gamma$ increased. $\theta$ for the more hydrophobic substrate was predicted accurately from the experimental water- $\mathrm{CO}_{2} \gamma$ and an interfacial model which only included long-ranged forces. However, for the more hydrophilic substrate, short-ranged specific interactions due to capping of the silanol groups by physisorbed $\mathrm{CO}_{2}$ resulted in an unusually large increase in the water-substrate $\gamma$ which led to a much larger increase in $\theta$ than predicted by the model. A novel type of $\theta$ hysteresis was discovered in which larger $\theta$ s were observed during depressurization relative to pressurization even down to ambient pressure. Effective receding angles were observed upon pressurization and effective advancing angles upon depressurization on the basis of movement of the three phase contact line. The greater degree of hysteresis for the more hydrophilic silica may be attributed in part to the capping of silanol groups with $\mathrm{CO}_{2}$. The large effects of $\mathrm{CO}_{2}$ on the various interfacial energies plays a key role in the enhanced ability of $\mathrm{CO}_{2}$, relative to many organic solvents, to dry silica surfaces as reported previously on the basis of FTIR spectroscopy.(Tripp, C.P. and Combes, J.R. Langmuir 1998, 14, 7348-7352).

4. Electrostatic Stabilization of Colloids in Carbon Dioxide: Electrophoresis and Dielectrophoresis

Over the last decade, steric stabilization has been achieved for a variety of inorganic and organic colloids in supercritical fluid carbon dioxide $\left(\mathrm{scCO}_{2}\right)$. Herein we demonstrate colloids may also be stabilized in $\mathrm{CO}_{2}$ by electrostatic forces, despite the ultra low dielectric constant of 
1.5. Zeta potentials of micron-sized water droplets, measured in a microelectrophoresis cell, reached -70mV corresponding to a few elementary charges per square micrometer of droplet surface. This degree of charge was sufficient to stabilize water/ $\mathrm{CO}_{2}$ emulsions for an hour, even with water volume fractions of 5\%. Hydrogen ions partition preferentially, relative to bicarbonate ions, from the emulsion droplets to the cores of surfactant micelles in the diffuse double layer surrounding the droplets. The micelles, formed with a low molecular weight branched hydrocarbon surfactant, prevent ion pairing of the hydrogen counterions to the negatively charged emulsion droplets. Dielectrophoresis of the water droplets at a frequency of $60 \mathrm{~Hz}$ leads to chains containing a dozen droplets with lengths of $50 \mu \mathrm{m}$. The ability to form electrostatically stabilized colloids in carbon dioxide is particularly useful in practical applications, since steric stabilization in $\mathrm{CO}_{2}$ is often limited by the poor solvation of the stabilizers.

5. Long-Ranged Electrostatic Repulsion and Crystallization of Emulsion Droplets in an Ultralow Dielectric Medium Supercritical Carbon Dioxide

Electostatic repulsion stabilizes micrometer-sized water droplets with spacings greater than $10 \mu \mathrm{m}$ in an ultra-low dielectric medium, $\mathrm{CO}_{2}(\varepsilon=1.5)$ at elevated pressures. The morphology of the water/ $\mathrm{CO}_{2}$ emulsion is characterized by optical microscopy and laser diffraction as a function of height. The counterions, stabilized with a nonionic highly branched stubby hydrocarbon surfactant, form an extremely thick double layer with a Debye screening length of $8.9 \mu \mathrm{m}$. As a result of the balance between electrostatic repulsion and the downwards force due to gravity, the droplets formed a hexagonal crystalline lattice at the bottom of the highpressure cell with spacings of over $10 \mu \mathrm{m}$. The osmotic pressure, calculated by solving the Poisson-Boltzmann equation, in the framework of the Wigner-Seitz cell model, is in good agreement with that determined from the sedimentation profile measured by laser diffraction. Thus, the long range stabilization of the emulsion may be attributed to electrostatic stabilization. The ability to form new types of colloids in $\mathrm{CO}_{2}$ with electrostatic stabilization is beneficial, since steric stabilization is often unsatisfactory due to poor solvation of the stabilizers. 
6. Water-in-Carbon dioxide Emulsions Stabilized with Hydrophobic Silica Particles

W/C emulsions were stabilized using hydrophobic silica particles adsorbed the interface, resulting in average droplet diameters as low as $7.5 \mu \mathrm{m}$. A porous cross-linked shell was formed about a hydrophilic (colloidal and fumed) silica core with a trifunctional silylating agent, (heptadecafluoro-1,1,2,2-tetrahydrodecyl)triethyoxy silane, to render the particles $\mathrm{CO}_{2}$ philic. The stability of emulsions comprising equal weights of $\mathrm{CO}_{2}$ and water was assessed with visual observations of settling fronts and the degree of emulsion coalescence, and the average drop size was measured by optical microscopy. The effect of $\mathrm{CO}_{2}$ density on both emulsion stability and droplet size was determined quantitatively. The major destabilizing mechanism of the emulsions was settling, whereas Ostwald ripening and coalescence were not visible at any density, even over 7 days. Flocculation of the settling droplets did not occur, although gelation of the emulsions through particle interactions resulted after longer periods of time. $\mathrm{CO}_{2}$-philic particles offer a new route to highly stable W/C emulsions, with particle energies of attachment on the order of $10^{6} \mathrm{kT}$, even at $\mathrm{CO}_{2}$ densities as low as $0.78 \mathrm{~g} / \mathrm{ml}$. At these low densities, surfactants rarely stabilize emulsions as the result of poor surfactant tail solvation. Recently, we have started a new project to extend this work for $\mathrm{CO}_{2}$ enhanced oil recovery, funded by Chevron.

7. Effect of Surface Hydrophilicity on Charging Mechanism of Colloids in Low-Permittivity Solvents

Electrophoretic mobilities of $\mathrm{TiO}_{2}$ colloids in an apolar solvent, toluene, were measured by differential-phase optical coherence tomography (DP-OCT). An electrode spacing of 0.18 mm, made possible by optical coherence tomography with transparent electrodes, enables measurement of the electrophoretic mobility with small samples (20 microliters) of highly turbid colloids at low applied electric potential to avoid electrohydrodynamic instability and electrochemical reactions. In the presence of Aerosol-OT reverse micelles, which stabilized the countercharges, the zeta potential was positive for hydrophilic $\mathrm{TiO}_{2}(13 \mathrm{mV}$ at $90 \mathrm{mM}$ AOT) and negative for hydrophobic $\mathrm{TiO}_{2}$. The magnitudes of the zeta potentials were very similar for 
these two types of $\mathrm{TiO}_{2}$ and decreased at the same rate with AOT concentration. For both hydrophilic and hydrophobic $\mathrm{TiO}_{2}$, a general mechanism is presented to describe the zeta potential in terms of preferential partitioning of cations and sulfosuccinate anions between the particle surface and reverse micelle cores in bulk. This preferential partitioning is governed by the hydrophilicities and extents of the particle surfaces and reverse micelle cores, as a function of surfactant and water concentration. The emerging understanding of the complex charging and stabilization mechanisms for colloids in apolar solvents will be highly beneficial for the design of novel materials.

\section{High Yield of Germanium Nanocrystals Synthesized from Germanium Diiodide in Solution}

High chemical yields, up to 73\%, were achieved for germanium $(\mathrm{Ge})$ nanocrystals synthesized in solution with germanium diiodide $\left(\mathrm{GeI}_{2}\right)$ and $\mathrm{LiAlH}_{4}$ as a reducing agent in tri-noctylphosphine (TOP) or tri-n-butylphosphine (TBP). Ge nanocrystals were characterized by transmission electron microscopy (TEM), energy dispersive X-ray spectroscopy (EDS), X-ray diffraction (XRD), Fourier transform infrared (FTIR) spectroscopy and X-ray photoelectron spectroscopy (XPS). Reactions in TOP at $300^{\circ} \mathrm{C}$ yielded Ge nanocrystals with moderate size polydispersity and good crystallinity with average diameters that could be manipulated by varying the precursor concentration from $\sim 3 \mathrm{~nm}$ to $\sim 11 \mathrm{~nm}$. High chemical yields are enabled by the high reactivity of $\mathrm{GeI}_{2}$, high $\mathrm{GeI}_{2}$ solubility in alkyl phosphines and relatively mild reaction temperature, which minimizes byproduct formation and solvent degradation. Compared to reactions carried out in TOP with the same concentration of $\mathrm{GeI}_{2}$, nanocrystals synthesized in $\mathrm{TBP}$ at $240^{\circ} \mathrm{C}$ exhibit larger size and broader size distribution. The presence of alkyl groups in the FTIR spectra, the small and controllable particle diameters and lack of significant Ge oxidation revealed by XPS indicate that the nanocrystals were chemically passivated with an organic layer.

9. Synthesis of Germanium Nanocrystals in High Temperature Supercritical CO2

Germanium nanocrystals were synthesized in supercritical (sc) $\mathrm{CO}_{2}$ by thermolysis of diphenylgermane (DPG) or tetraethylgermane (TEG) with octanol as a capping ligand at $500^{\circ} \mathrm{C}$ 
and 27.6 MPa. The Ge nanocrystals were characterized with high resolution transmission electron microscopy (HRTEM), energy-dispersive X-ray spectroscopy (EDS), and X-ray diffraction (XRD). On the basis of TEM, the mean diameters of the nanocrystals made from DPG and TEG were 10.1 and 5.6nm, respectively. The synthesis in sc- $\mathrm{CO}_{2}$ produced much less organic contamination compared with similar reactions in organic supercritical fluids. When the same reaction of DPG with octanol was performed in the gas phase without $\mathrm{CO}_{2}$ present, bulk Ge crystals were formed instead of nanocrystals. Thus, the solvation of the hydrocarbon ligands by $\mathrm{CO}_{2}$ was sufficient to provide steric stabilization. The presence of steric stabilization in $\mathrm{CO}_{2}$ at a reduced temperature of 2.5 , with a reduced solvent density of only 0.4 , may be attributed to a reduction in the differences between ligand-ligand interactions and ligand- $\mathrm{CO}_{2}$ interactions relative to thermal energy.

\section{References that acknowledge DOE BES support (published)}

Adkins SS, Gohil D, Dickson JL, Webber SE, Johnston KP. 2007. Water-in-carbon dioxide emulsions stabilized with hydrophobic silica particles. Physical Chemistry Chemical Physics 9(48):6333-6343.

Dhanuka VV, Dickson JL, Ryoo W, Johnston KP. 2006. High internal phase CO2-in-water emulsions stabilized with a branched nonionic hydrocarbon surfactant. Journal of Colloid and Interface Science 298(1):406-418.

Dickson JL, Adkins SS, Cao T, Webber SE, Johnston KP. 2006. Interactions of Core-Shell Silica Nanoparticles in Liquid Carbon Dioxide Measured by Dynamic Light Scattering. Ind. Engr. Chem. Res. 45(16):5603-5613.

Dickson JL, Gupta G, Horozov TS, Binks BP, Johnston KP. 2006. Wetting Phenomena at the CO2/Water/Glass Interface. Langmuir 22(5):2161-2170.

Dickson JL, Smith PG, Dhanuka VV, Srinivasan V, Stone MT, Rossky PJ, Behles JA, Keiper JS, $\mathrm{Xu} \mathrm{B}$, Johnson CS and others. 2005. Interfacial Properties of Fluorocarbon and Hydrocarbon Phosphate Surfactants at the Water - CO2 Interface. Industrial \& Engineering Chemistry Research 44:1370-1380.

Gupta G, Shah PS, Zhang X, Saunders AE, Korgel BA, Johnston KP. 2005. Enhanced Infusion of Gold Nanocrystals into Mesoporous Silica with Supercritical Carbon Dioxide. Chemistry of Materials 17(26):6728-6738.

Gupta G, Stowell CA, Patel MN, Gao X, Yacaman MJ, Korgel BA, Johnston KP. 2006. Infusion of Presynthesized Iridium Nanocrystals into Mesoporous Silica for High Catalyst Activity. Chem. Materials 18(26):6239-6249. 
Li Y, Meli L, Lim KT, Johnston KP, Green PF. 2006. Structural Inversion of Micellar Block Copolymer Thin Films. Macromolecules 39(20):7044-7054.

Li Y, Pham JQ, Johnston KP, Green PF. 2007. Contact Angle of Water on Polystyrene Thin Films: Effects of CO2 Environment and Film Thickness. Langmuir 23(19):9785-9793.

Lu X, Fanfair DD, Johnston KP, Korgel BA. 2005. High Yield Solution-Liquid-Solid Synthesis of Germanium Nanowires. Journal of the American Chemical Society 127(45):1571815719.

Lu X, Korgel BA, Johnston KP. 2005. High Yield of Germanium Nanocrystals Synthesized from Germanium Diiodide in Solution. Chemistry of Materials 17(25):6479-6485.

Lu X, Korgel BA, Johnston KP. 2005. Synthesis of germanium nanocrystals in high temperature supercritical CO2. Nanotechnology 16(7):389-394.

Patel MN, Williams RD, May RA, Uchida H, Stevenson KJ, Johnston KP. 2008. Electrophoretic Deposition of Au Nanocrystals inside Perpendicular Mesochannels of TiO2. Chemistry of Materials 20(19):6029-6040.

Ryoo W, Dickson JL, Dhanuka VV, Webber SE, Bonnecaze RT, Johnston KP. 2005. Electrostatic Stabilization of Colloids in Carbon Dioxide: Electrophoresis and Dielectrophoresis. Langmuir 21:5914-5923.

Ryoo W, Webber SE, Bonnecaze RT, Johnston KP. 2006. Long-Ranged Electrostatic Repulsion and Crystallization of Emulsion Droplets in an Ultralow Dielectric Medium Supercritical Carbon Dioxide. Langmuir 22(3):1006-1015.

Saunders AE, Dickson JL, Shah PS, Lee MY, Lim KT, Johnston KP, Korgel BA. 2006. Breath figure templated self-assembly of porous diblock copolymer films. Physical Review E: Statistical, Nonlinear, and Soft Matter Physics 73(3-1):031608/1-031608/7.

Smith PG, Jr., Dhanuka VV, Hwang HS, Lim KT, Johnston KP. 2007. Tertiary Amine Esters for Carbon Dioxide Based Emulsions. Industrial \& Engineering Chemistry Research 46(8):2473-2480.

Smith PG, Jr., Patel MN, Kim J, Johnston KP, Milner TE. 2007. Electrophoretic Mobility Measurement by Differential-Phase Optical Coherence Tomography. J. Phys. Chem. C 111(6):2614-2622.

Smith PG, Jr., Ryoo W, Johnston KP. 2005. Electrostatically Stabilized Metal Oxide Particle Dispersions in Carbon Dioxide. Journal of Physical Chemistry B 109(43):20155-20165.

Smith PGJr, Patel MN, Kim J, Milner TE, Johnston KP. 2007. Effect of Surface Hydrophilicity on Charging Mechanism of Colloids in Low-Permittivity Solvents. J. Phys. Chem. C 111(2):840-848. 
Zhang X, Johnston KP. 2007. Supercritical CO2-based solvents in next generation microelectronics processing. Chin. Sci. Bull. 52(1):27-33. 